\title{
Welchen Nutzen bieten Businessplan-Wettbewerbe für Gründungsvorhaben?
}

\author{
Prof. Dr. Dodo zu Knyphausen-Aufseß, Berlin, und Dipl.-Kffr. Lea Stadtler, Genf
}

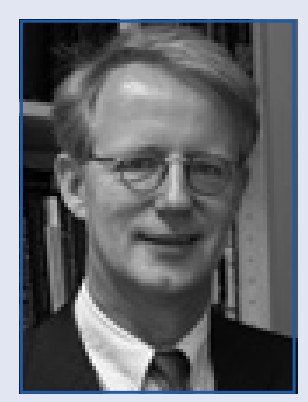

Prof. Dr. Dodo zu Knyphausen-Aufseß ist Leiter des Fachgebietes Strategische Führung und Globales Management an der Technischen U. Berlin. Bevorzugte Forschungsgebiete: Strategische Unternehmensführung, Diversifikation, Mergers\&Acquisitions.

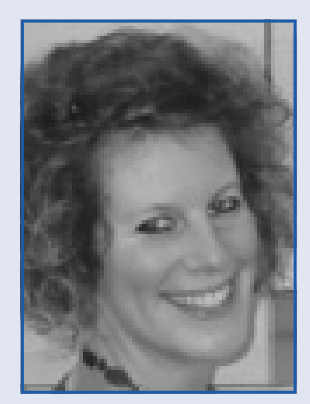

Dipl.-Kffr. Lea Stadtler ist wissenschaftliche Mitarbeiterin am Lehrstuhl für Organisation und Management, HEC an der Universität Genf. Bevorzugte Forschungsgebiete: Public-private Partnerships, Organisation und Change Management.

Zur Analyse des Nutzens von Businessplan-Wettbewerben (BPW) lassen sich in Anlehnung an die Wettbewerbsliteratur zwei Funktionen - die Selektion- und die Förderfunktion - ableiten. Auf Basis von 22 empirischen Studien zur Gründungsplanung zeigt sich, dass BPW, die allein auf die Bewertung von Businessplänen, nicht aber auf weitere Unterstützungsangebote fokussieren, zwar die Selektionsfunktion ausüben können, im Hinblick auf die Förderfunktion jedoch Defizite aufweisen. Vor diesem Hintergrund wird ein theoretischer Bezugsrahmen zur Analyse des Nutzens weiterer Unterstützungsangebote von BPW entwickelt.

Stichwörter: Businessplan-Wettbewerbe, Business Planning, Start-ups, Unternehmensgründung, Gründungserfolg

\section{Konzeption und Ziele von Businessplan- Wettbewerben}

Das Konzept der Businessplan-Wettbewerbe (BPW) zur Stärkung der Gründungskultur und Unterstützung von Gründungsvorhaben hat seit seiner erstmaligen Durchführung in Deutschland im Jahre 1996 verstärkt an Bedeutung gewonnen. Die Forschung in Bezug auf den Nutzen und die Gestaltung von BPW befindet sich jedoch noch in den Anfängen (vgl. Dippe/Müller, 2005; Chwolka/Raith, 2008; zu Knyphausen-Aufsess/Goodwin, 2008), und entsprechend gibt es auch nur wenige praktische Hilfestellungen für die Organisatoren von und die Teilnehmer an solchen Wettbewerben.

BPW gibt es mit unterschiedlich ausgestalteten Rahmenprogrammen; ihnen gemeinsam ist allerdings, dass sie die Erstellung von Businessplänen (BP) zur Unternehmensgründung begleiten und prämieren. Die Erstellung von BP ist eine spezielle, d. h. gründungsbezogene Form der strategischen Planung, deren Wert in der akademischen Literatur umstritten ist. Wenn aber das Kernelement der BPW - Erstellung eines BP - hinsichtlich seines Nutzens zweifelhaft ist, muss man sich die Frage stellen, warum es BPW überhaupt gibt und warum diese bspw. durch staatliche Institutionen unterstützt werden sollten. Ist eine Teilnahme für Gründer empfehlenswert? Sind die durch BPW generierten Start-ups besser aufgestellt und somit interessant für Venture-Capital-Gesellschaften oder andere Eigenkapitalgeber?

Im Folgenden wird der Nutzen von BPW untersucht, um damit mögliche Antworten auf diese und ähnliche Fragen zu liefern. Es werden zunächst (in umgekehrter Reihenfolge) die beiden Begriffsbestandteile „Businessplan“ und „Wettbewerb“ analysiert, bevor ein theoretischer Bezugsrahmen entwickelt und darauf bezogene Propositionen präsentiert werden.

\section{Funktionen von „Wettbewerb”}

In Bezug auf den Nutzen von BPW für Gründungsvorhaben können zwei Funktionen unterschieden werden. Zum einen schaffen BPW einen ,Wettbewerb vor dem Wettbewerb“, indem Geschäftskonzepte beurteilt, die besten nominiert und mit Preisen ausgezeichnet werden. Preise fungieren als das knappe Gut, wodurch bereits vor der Marktpenetration zusätzliche Leistungsanreize gesetzt und eine vorgezogene Selektion durchgeführt werden. Die Selektion beschränkt sich jedoch nicht nur auf die Nominierung. Die Teilnehmer bekommen auf Basis ihres BP ein Expertenfeedback über die Erfolgschancen des Gründungsvorhabens. Das Feedback, zusammen mit den im Verlaufe des Wettbewerbs gewonnenen Erkenntnissen über Stärken und Schwächen, ermöglicht eine fundierte 
Entscheidung, ein nicht geeignetes Vorhaben gegebenenfalls noch in der Anfangsphase abzubrechen. Diese Selektion ist durchaus positiv zu beurteilen, da sowohl der potenzielle Schaden für den gescheiterten Gründer (z. B. investierte Zeit, Motivation und Geld) als auch für die Volkswirtschaft insgesamt (z. B. potenzielle Vertragsbrüche wegen Insolvenz) begrenzt wird.

Neben der Selektion geht es zum anderen jedoch auch um die Förderung aussichtsreicher Unternehmen im Hinblick auf ihren Aufbau und die Etablierung am Markt. In Analogie zu der unterstützenden Wettbewerbspolitik des Staates haben sich die Träger von BPW die Unterstützung von Gründern in der Vor-Wettbewerbsphase zur Aufgabe gemacht. Durch das Angebot eines umfangreichen Rahmenprogramms werden den Teilnehmern gleiche Chancen für die Optimierung der Idee und dessen Umsetzung gewährt.

Im Ergebnis ergeben sich aus der wettbewerbstheoretischen Perspektive also zwei zentrale Funktionen von BPW:

1. Ausfilterung der nicht Erfolg versprechenden Projekte;

2. Förderung der Erfolg versprechenden Projekte.

\section{Nutzen von „Businessplänen“}

Eine Unternehmensgründung ,,als Schaffung eines auch in Zukunft tragfähigen und flexiblen Fundaments umfasst markt-, produkt- und/oder verfahrensbezogene Entscheidungen“" (Szyperski/Nathusius, 1977, S. 24). Die Komplexität dieser Entscheidungsprozesse, bedingt zum Beispiel durch die Notwendigkeit der Bildung von Annahmen über die Entwicklungen unsicherer und volatiler Umwelten, eine meist stark eingeschränkte Ressourcenausstattung, fehlende Erfahrungswerte sowie ein hohes Legitimationsbedürfnis, spricht für einen gesonderten Untersuchungsgegenstand innerhalb der Forschung zur strategischen Planung. Der Nutzen des BP, als formalisiertes Ergebnis des Business Planning, wird in der Gründungsliteratur dabei kontrovers diskutiert.

Die Argumente für die Erstellung eines BP lassen sich nach der zeitlichen Wirkung des Effekts (vor bzw. nach Marktpenetration) unterscheiden. Für die Bestimmung des Nutzens noch vor der Marktpenetration kann Rückgriff auf die allgemeine Planungsliteratur genommen werden, der zufolge die Planung der Antizipation von Problemen der Steigerung der Motivation durch Formulierung klarer und herausfordernder Ziele sowie der Orientierung und Priorisierung im Ressourcenmanagement dienen kann (vgl. Mintzberg, 1995; Castrogiovanni, 1996).

Nach Marktpenetration kann das Gründerteam von seiner Planungserfahrung profitieren und die Planungsergebnisse zur Koordination sowie zur internen und externen Kommunikation nutzen; damit dürfte sich dann auch die Effizienz der Leistungserstellung verbessern. In der Konsequenz hilft ein Business Planning der Chancenwahrnehmung und der Verbesserung der Chancenrealisierung.
Gegen ein Business Planning wird bereits vor Markteintritt die Bedeutung der Intuition zur Chancenwahrnehmung und Marktbearbeitung angeführt; das Business Planning sei dann nur eine Ressourcen- und Zeitverschwendung. Das oft unsichere und innovative Umfeld senke die Prognosegüte der Planungsprämissen und damit den Planungsnutzen nach Markteintritt. Darüber hinaus könne die Planung zu sinnlosen Ritualen sowie dem deterministischen Umgangs mit Planungsergebnissen führen. Gemäss dieser Argumentation reduziert ein Business Planning die Chancenwahrnehmnung und ist somit durch Tätigkeiten aktiver Umweltbeeinflussung zu ersetzen (vgl. Baker/Nelson, 2005, S. 335 f.).

Zur Untersuchung empirischer Befunde zum Business Planning haben die Autoren eine Literaturrecherche der Datenbanken „Abi/Inform“ und „EbscoHost“ mit den Suchbegriffen „,business plan“, „,business planning“ und „start-up“ durchgeführt. Ein Rückgriff auf angegebene Referenzen ermöglichte zudem eine Erhöhung der Anzahl der als relevant eingestuften Studien. Für den Einbezug einer Studie in die Analyse ist dabei entscheidend, dass sie den Effekt einer gründungsbezogenen Planung misst. Im Ergebnis konnten 22 Studien identifizieren werden. Die selektierten Studien unterscheiden sich darin, ob der Nutzen des BP vor oder nach Markeintritt gemessen wurde, sowie in den verwendeten Erfolgsvariablen (vgl. Tab. 1).

Die Studien unterstützen insgesamt die Annahme, dass ein Business Planning dazu führt, Erfolg versprechende Projekte schneller zu realisieren und aussichtslose Projekte schneller abzubrechen. Bei der Messung des Planungserfolgs nach dem Markteintritt ergeben sich jedoch uneinheitliche Ergebnisse, die sowohl Pro- als auch Kontra-Argumente unterstützen. Es wird vermutet, dass der Planungsnutzen von der Gestaltung des Planungsprozesses und -inhalts abhängt. Das optimale Planungs-Timing hängt zum Beispiel von der Wahrnehmung der finanziellen und wettbewerbspolitischen Umwelt $a b$. Eine umfangreiche, detaillierte Planung zeigt sich als weniger geeignet in dynamischen Umwelten sowie in frühen Phasen der Unternehmensgründung, wohin gegen sie sich sowohl in komplexen Umwelten als auch in späteren Entwicklungsphasen als vorteilhaft erweist.

Darüber hinaus ist der Grad der optimalen Formalisierung von der gewählten Strategie und der Managementerfahrung abhängig. Letztlich beeinflusst der kulturelle Hintergrund das Bedürfnis der Unsicherheitsvermeidung und damit auch den Umgang mit den Planungsergebnissen. Studienergebnisse bestätigen einen negativen Einfluss eines hohen Unsicherheitsvermeidungsbedürfnisses, der sich durch ein mögliches krampfhaftes Festhalten an Planungsergebnissen seitens der Gründer und damit einer Reduktion an Flexibilität und Planungsanpassungen erklären lässt.

Trotz dieser sehr differenzierten Befunde lassen sich auch einige allgemeine Empfehlungen ableiten: Eine Grobplanung bereits vor dem ersten Kundenkontakt, dem Res- 


\begin{tabular}{|c|c|c|}
\hline Autor(en) & Ergebnisse & $\begin{array}{l}\text { Erfolgs- } \\
\text { variable }\end{array}$ \\
\hline \multicolumn{3}{|c|}{ Messung in der Gründungsphase } \\
\hline $\begin{array}{l}\text { Liao/Gartner, 2007/08 } \\
\quad \text { (Journal of Small } \\
\text { Business Strategy) }\end{array}$ & $\begin{array}{l}\text { Mit der Erstellung des BP steigt die Gründungswahrscheinlichkeit um den } \\
\text { Faktor } 6 .\end{array}$ & Start-up Status \\
\hline $\begin{array}{l}\text { Reynolds, } 2007 \\
\text { (Foundation and Trends } \\
\text { in Entrepreneurship) }\end{array}$ & $\begin{array}{l}\text { Die Planung als Teil des Gründungsprozesses beeinflusst die Entscheidung } \\
\text { zur Realisation der Gründungsidee. Dies führt bei positiver Entscheidung zu } \\
\text { einem schnelleren Gründungsprozess und zu einem schnellen Abbruch bei } \\
\text { negativem Entschluss. }\end{array}$ & $\begin{array}{l}\text { Status: Am Markt, in } \\
\text { Gründung, Abbruch }\end{array}$ \\
\hline $\begin{array}{l}\text { Liao/Gartner, } 2006 \\
\text { (Small Business } \\
\text { Economics) }\end{array}$ & $\begin{array}{l}\text { Mit der Erstellung eines BP steigt die Wahrscheinlichkeit der Fortsetzung } \\
\text { der Gründungsaktivitäten um den Faktor } 2,6 \text {. } \\
\text { Die Wahrscheinlichkeit der Gründungsfortführung steigt } \\
\text {-mit einer frühzeitigen Planung in finanziell und wettbewerbsbezogen unsi- } \\
\text { cheren Umwelt. } \\
\text {-mit einer späten Planung in als finanziell und wettbewerbsbezogen sicher } \\
\text { wahrgenommenen Umwelt. }\end{array}$ & $\begin{array}{l}\text { Fortführung der } \\
\text { Gründungsaktivitäten }\end{array}$ \\
\hline $\begin{array}{l}\text { Delmar/Shane, } 2004 \\
\text { (Journal of Business } \\
\text { Venturing) }\end{array}$ & $\begin{array}{l}\text { Für Gründer, die ein BP durchgeführt haben, ist die Wahrscheinlichkeit (1) } \\
\text { geringer, das Vorhaben während der ersten } 3 \text { Jahre aufzugeben und (2) hö- } \\
\text { her, die Produktentwicklung, Marketingaktivitäten und den Ressourcener- } \\
\text { werb zu realisieren. }\end{array}$ & Abbruch \\
\hline $\begin{array}{l}\text { Shane/Delmar, } 2004 \\
\text { (Journal of Business } \\
\text { Venturing) }\end{array}$ & $\begin{array}{l}\text { Gründungsvorhaben werden weniger oft abgebrochen sofern vor den ersten } \\
\text { Marketingaktivitäten und Kundenkontakt ein BP erstellt wurde. }\end{array}$ & Abbruch \\
\hline $\begin{array}{l}\text { Delmar/Shane, } 2003 \\
\text { (Strategic Management } \\
\text { Journal) }\end{array}$ & $\begin{array}{l}\text { Business Planning reduziert die Wahrscheinlichkeit des Abbruchs um knapp } \\
60 \% \text { und erleichtert die Produktentwicklung und Organisation. }\end{array}$ & $\begin{array}{l}\text { Produktentwicklung, } \\
\text { Organisation, Abbruch }\end{array}$ \\
\hline \multicolumn{3}{|c|}{ Messung in der Bewährungsphase } \\
\hline $\begin{array}{l}\text { Kraus/Schwarz, } 2007 \\
\text { (International Journal of } \\
\text { Management and Enter- } \\
\text { prise Development) }\end{array}$ & Signifikante, positive Beziehung zwischen BP und Unternehmenserfolg & $\begin{array}{l}\text { Wachstum der } \\
\text { Mitarbeiterzahl }\end{array}$ \\
\hline $\begin{array}{l}\text { Herr, } 2007 \\
\text { (Dissertation, } \\
\text { Wiesbaden) }\end{array}$ & $\begin{array}{l}\text { Signifikante, positive Beziehung zwischen BP und Umsatz im lfd. Ge- } \\
\text { schäftsjahr; keine Signifikanz bei weiteren Erfolgsmaßen }\end{array}$ & $\begin{array}{l}\text { Zufriedenheit bzgl. } \\
\text { Umsatz, Mitarbeiter, } \\
\text { Ergebnis }\end{array}$ \\
\hline $\begin{array}{l}\text { Gruber, } 2007 \\
\text { (Journal of Business } \\
\text { Venturing) }\end{array}$ & $\begin{array}{l}\text { Der Nutzen der Planung variiert mit (1) Art des Gründungsumfeld (2) Art } \\
\text { der Planungsaktivität (3) Planungsanstrengung } \rightarrow \text {,trade-off regime“ in stark } \\
\text { dynamischem Umfeld, „munificient regime“ in konsistenterem Umfeld } \\
\text { Erfolg versprechender }\end{array}$ & $\begin{array}{l}\text { Subjektive Erreichung } \\
\text { der Zielerreichung im } \\
\text { Marketing }\end{array}$ \\
\hline $\begin{array}{l}\text { Lange et al., } 2007 \\
\text { (Venture Capital) }\end{array}$ & $\begin{array}{l}\text { Kein signifikanter Unterschied zwischen Erfolg von Unternehmen mit bzw. } \\
\text { ohne BP }\end{array}$ & $\begin{array}{l}\text { Einnahmen, Nettoge- } \\
\text { winn, Mitarbeiterzahl }\end{array}$ \\
\hline $\begin{array}{l}\text { Honig/Karlsson, } 2004 \\
\text { (Journal of Management) }\end{array}$ & $\begin{array}{l}\text { Nicht signifikante Erhöhung der Überlebenschance um Faktor } 1,8 \text { bei Vor- } \\
\text { liegen eines BP; positiver aber ebenfalls nicht signifikanter Effekt auf die } \\
\text { Rentabilität. }\end{array}$ & Überleben, Rentabilität \\
\hline $\begin{array}{l}\text { Bhidé, } 2000 \\
\text { (Buchveröffentlichung, } \\
\text { Oxford) }\end{array}$ & $\begin{array}{l}\text { Kein Zusammenhang zwischen Start-up Erfolg und intensiver Planung. } \\
\text { Bei den erfolgreichen Firmen wird wenig Zeit für die Gründungsvorberei- } \\
\text { tung aufgewandt. Unter den Planern hat die Mehrheit lediglich rudimentäre } \\
\text { Pläne. }\end{array}$ & Umsatzwachstum \\
\hline $\begin{array}{l}\text { Van Gelderen/Frese/ } \\
\text { Thurik, } 2000 \\
\text { (Small Business } \\
\text { Economics) }\end{array}$ & $\begin{array}{l}\text { Die Dynamik der Umwelt beeinflusst den Wert einer umfassenden Planung. } \\
\text { Aus diesem Grund ist eine punktuelle Planung in der ersten Lebensphase er- } \\
\text { folgreich, in späteren Lebensphasen ist jedoch eine umfangreichere Planung } \\
\text { erfolgreicher. }\end{array}$ & $\begin{array}{l}\text { Wirtschaftlicher und } \\
\text { persönlicher Erfolg }\end{array}$ \\
\hline
\end{tabular}

Tab. 1: Studien zum Business Planning 


\begin{tabular}{|c|c|c|}
\hline Autor(en) & Ergebnisse & $\begin{array}{l}\text { Erfolgs- } \\
\text { variable }\end{array}$ \\
\hline $\begin{array}{l}\text { Rauch/Frese, } 1998 \\
\text { (www.babson.edu) }\end{array}$ & $\begin{array}{l}\text { In stark unsicheren und wettbewerbsintensiven Umwelten sind Gründer, die } \\
\text { intensiv planen erfolgreicher als diejenigen, die wenig planen. In sicheren, } \\
\text { wenig wettbewerbsintensiven Umwelten sind Gründer erfolgreicher, die } \\
\text { wenig planen. }\end{array}$ & $\begin{array}{l}\text { Wachstum, Größe, } \\
\text { Einkommen, Zufrie- } \\
\text { denheit, Selbstbewer- } \\
\text { tung }\end{array}$ \\
\hline $\begin{array}{l}\text { Lumpkin et al., } 1998 \\
\text { (www.babson.edu) }\end{array}$ & $\begin{array}{l}\text { Sehr schwacher Zusammenhang zwischen Business Planning und Perfor- } \\
\text { mance allgemein. Die Eignung bestimmter Planungsinhalte variiert mit dem } \\
\text { Unternehmensstadium, die Eignung einer Formalisierung variiert je nach } \\
\text { Planungsinhalt. }\end{array}$ & Konkurrenzvergleich \\
\hline $\begin{array}{l}\text { Brüderl et al., } 1996 \\
\text { (Buchveröffentlichung, } \\
\text { Berlin) }\end{array}$ & $\begin{array}{l}\text { Die Erstellung eines BP erhöht die Wahrscheinlichkeit des Umsatzwachs- } \\
\text { tums. }\end{array}$ & $\begin{array}{l}\text { Survival, Beschäfti- } \\
\text { gungszuwachs, Um- } \\
\text { satzwachstum }\end{array}$ \\
\hline $\begin{array}{l}\text { Olson/Bokor, } 1995 \\
\text { (Journal of Small } \\
\text { Business Management) }\end{array}$ & $\begin{array}{l}\text { Der Erfolg von kleinen, schnell wachsenden Unternehmen ist beeinflusst } \\
\text { von der Interaktion von Planungsformalität und Planungsinhalt. Der optima- } \\
\text { le Formalisierungsgrad ist abhängig von (1) gewählten Strategie und (2) } \\
\text { Erfahrung des Managements. }\end{array}$ & Umsatzwachstum \\
\hline $\begin{array}{l}\text { Duchesneau/Gartner, } \\
1990 \text { (Journal of } \\
\text { Business Venturing) }\end{array}$ & $\begin{array}{l}\text { Erfolgreiche Firmen: (1) verwenden einen Planungsprozess ähnlich dem } \\
\text { PPM (2) planen länger ( } 237 \text { vs. } 85 \text { Std.) (3) verfolgen wenig Markt-/ } \\
\text { Wettbewerberanalyse, (jedoch mehr als nicht erfolgreiche Firmen) (4) } \\
\text { nutzen Informationen kritisch zur Verbesserung (5) nutzen die schriftliche } \\
\text { Fixierung vereinzelt. }\end{array}$ & Finanzielles Ergebnis \\
\hline $\begin{array}{l}\text { Shuman et al., } 1985 \\
\text { (Long Range Planning) }\end{array}$ & $\begin{array}{l}\text { 95,5\% der Unternehmen ohne BP sind profitabel gegenüber nur } 84,1 \% \text { der } \\
\text { Unternehmen mit BP (Aufschlüsselung der Unternehmen mit Gewinnmarge } \\
\text { über 16\%: 19,8\% der Unternehmen mit BP, 30\% der Unternehmen ohne BP). }\end{array}$ & $\begin{array}{l}\text { Gewinnmarge, } \\
\text { subjektive Nutzen- } \\
\text { einschätzung }\end{array}$ \\
\hline $\begin{array}{l}\text { Robinson et al., } 1984 \\
\quad \text { (Journal of Small } \\
\text { Business Management) }\end{array}$ & $\begin{array}{l}\text { Bereits geringe Planung erzeugt eine positive Performance Wirkung, inten- } \\
\text { sivere Planungsaktivitäten variieren in ihrer Wirkweise. }\end{array}$ & $\begin{array}{l}\text { Absatzwachstum, } \\
\text { Rentabilität, Absatz } \\
\text { pro Mitarbeiter, Mitar- } \\
\text { beiterzahl }\end{array}$ \\
\hline $\begin{array}{l}\text { Van de Ven et al., } 1984 \\
\text { (Journal of Management) }\end{array}$ & $\begin{array}{l}\text { Der Planungsprozess erfolgreicher Firmen ist geprägt von: } \\
\text { (1) der Anwendung des „Program Planning“-Modells (mit den Phasen (a) } \\
\text { Problem Exploration, (b) Knowledge Exploration, (c) BP Development, (d) } \\
\text { Company Startup), (2) lediglich punktueller Formalisierung, (3) Einbindung } \\
\text { von Außenstehenden. } \\
\text { Der BP erfolgreicher Firmen (1) wird stärker verbreitet (z. B. an Investoren } \\
\text { geschickt) (2) wird proaktiv zur Kundenansprache benutzt. }\end{array}$ & $\begin{array}{l}\text { Wachstum, Abhängig- } \\
\text { keit, subjektive } \\
\text { Erfolgsbewertung }\end{array}$ \\
\hline \multicolumn{3}{|c|}{ Metastudie } \\
\hline $\begin{array}{l}\text { Brinckmann/Grichnik/ } \\
\text { Kapsa, } 2010 \\
\text { (Journal of Business } \\
\text { Venturing) }\end{array}$ & $\begin{array}{l}\text { Zwischen der Planung und dem Erfolg besteht ein schwacher positiver Zu- } \\
\text { sammenhang. Kontextfaktoren, wie bspw. das kulturelle Umfeld, beeinflus- } \\
\text { sen den Zusammenhang. }\end{array}$ & $\begin{array}{l}\text { Wachstum, Ertrags- } \\
\text { kraft, Überleben }\end{array}$ \\
\hline
\end{tabular}

Tab. 1 (Fortsetzung)

sourcenerwerb und der Produktentwicklung erweist sich als hilfreich. Während der Planung sind bestimmte Elemente wie der Kundennutzen und die Finanzierung zu fokussieren, während angesichts der Ressourcenknappheit in der Gründungsphase anderen Elementen (z. B. Markt- und Wettbewerbsanalyse) weniger Energie gewidmet werden kann. Zudem ist ein einfaches Prozessdesign vorteilhaft (z. B. Problemuntersuchung, Wissensanalyse, BP-Erstellung und kontinuierliche Anpassung), bei dem insbesondere Sekundärinformationen verwendet werden sollten. Um den Zeit- und Ressourcenaufwand zu reduzieren, empfiehlt sich bei der Formalisierung die Fokussierung auf Kernelemente.
Wichtig ist in diesem Zusammenhang auch, dass Planung und Ausführung simultan ausgeführt und FeedbackSchleifen zugelassen werden (vgl. Brinckmann et al., 2010). Als Referenz für diesen Prozess kann das von McGrath/MacMillan (1995) entwickelte „Discovery-driven Planning"-Modell herangezogen werden. Der Schwerpunkt liegt hierbei auch auf der kontinuierlichen Überprüfung getroffener Annahmen mittels eingebauter Meilensteine, die letztlich auch der Sicherung der Flexibilität dient. Abschliessend ist festzuhalten, dass sich der Einbezug Außenstehender in die Planung sowie die Nutzung der Planungsergebnisse zur internen und externen Kommunikation als vorteilhaft erweisen. 
Die Ergebnisse geben insgesamt Hinweise dazu, dass ein Business Planning nicht prinzipiell, sondern nur bei individueller Anpassung an die Gründungskonditionen zu einem positiven Nutzen führen kann. Diese Vermutung wird von einer Meta-Analyse (vgl. Brinckmann et al., 2010) unterstützt, die einen positiven, jedoch relativ schwachen $\mathrm{Zu}$ sammenhang von Planung und Erfolg konstatiert, der stark von Moderatoren abhängig ist. In Konsequenz bedeutet dies für BPW, dass die Forderung und Prämierung von BP zwar der Ausübung der Selektionsfunktion dienlich ist; ein Nutzen in Bezug auf die Förderfunktion ist jedoch nur bedingt gegeben. Somit ist der Mehrwert derjenigen BPW, die lediglich die Erstellung eines BP fordern und prämieren, limitiert. In Deutschland, der Schweiz und Österreich haben sich jedoch zunehmend BPW mit einem umfangreichen Rahmenprogramm etabliert, das Angebote wie Workshops, Coaching und Netzwerkveranstaltungen umfasst.

\section{Entwicklung eines Bezugsrahmens und Ableitung von Propositionen}

Wie wirken BPW mit ihrem gesamten Rahmenprogramm auf die Gründung und Etablierung von jungen Unternehmen? In Anlehnung an Dippe/Müller (2005) kann zur Erstellung eines Bezugsrahmens zur Beantwortung dieser Frage Rückgriff auf theoretische Ansätze zum Gründungs- prozess, insbesondere auf die personen-, umfeld-, sowie unternehmensbezogenen Ansätze genommen werden. Interpretiert man den Gründungsprozess in Anlehnung an den ressourcenorientierten Ansatz als einen Prozess der Ressourcenakkumulation, in dem die anfänglichen persönlichen Ressourcen erweitert und sinnvoll durch externe Ressourcen ergänzt werden, ergibt sich eine Verbindung der oben genannten Ansätze. Dabei sind die personenund umfeldbezogenen Ansätze auf die Art der Ressourcenquelle bezogen und der unternehmensbezogene Ansatz bildet die dynamische Komponente der Ressourcenakkumulation.

Den Ausgangspunkt des sich ergebenden Bezugsrahmens (vgl. $A b b .1$ ) bilden die Unterstützungsleistungen von BPW. Sie werden danach eingeordnet,

(1) an welcher Ressourcenquelle,

(2) in welcher Phase im Gründungsprozess sowie

(3) an welcher erfolgsrelevanten Ressource sie ansetzen.

Anhand dieses Bezugsrahmens lassen sich Propositionen ableiten (vgl. Tab. 2), die darstellen, welchen Beitrag BPW durch bestimmte Unterstützungsleistungen für den Gründungserfolg liefern können.

Die Propositionen sind den vier Phasen, und zwar der Sensibilisierungs-, der Vorbereitungs-, der Errichtungs- und der Bewährungsphase zugeordnet. Darüber hinaus werden

\begin{tabular}{|c|c|c|c|c|c|c|c|c|c|c|c|c|}
\hline $\begin{array}{l}\text { Person } \\
\text { (Personen- } \\
\text { bezogener } \\
\text { Ansatz) }\end{array}$ & $\begin{array}{l}\text { Öffentlichkeits- } \\
\text { arbeit } \\
\rightarrow \text { Gründungs- } \\
\text { einstellung } \\
\text { (Gründungs- } \\
\text { klima) }\end{array}$ & \multicolumn{3}{|c|}{$\begin{array}{l}\text { KEINE Unterstützung } \\
\rightarrow \text { Allgemeinbildung } \\
\rightarrow \text { Innovationskraft } \\
\rightarrow \text { fachbezogene } \\
\text { Fähigkeiten } \\
\rightarrow \text { Proaktive } \\
\text { Entwicklungsfähigkeit } \\
\qquad \mid\end{array}$} & \multicolumn{5}{|c|}{ 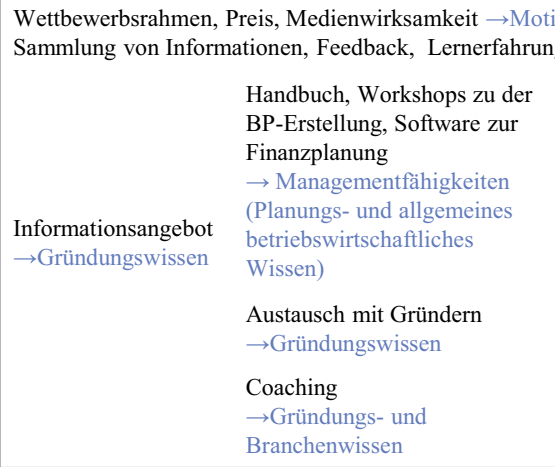 } & \multicolumn{2}{|c|}{$\begin{array}{l}\text { ation, Teamarbeit } \\
\text { Semïsentation } \rightarrow \text { Unsicherheits } \\
\rightarrow \text { Managementfähigkeiten } \\
\text { (insb. Kommunikations- } \\
\text { und Präsentations- } \\
\text { techniken, Rechtswissen, } \\
\text { kaufmännisches Knowhow) }\end{array}$} & $\begin{array}{l}\text { Seminare } \\
\text { Beratung und } \\
\text { Mentoring } \\
\rightarrow \text { Branchenwissen } \\
\rightarrow \text { Management- } \\
\text { fähigkeiten }\end{array}$ \\
\hline \begin{tabular}{|c} 
Idee \\
(Unterneh- \\
mensbezogener \\
Ansatz)
\end{tabular} & & \multicolumn{3}{|c|}{$\begin{array}{l}\rightarrow \text { Gründungsidee mit } \\
\text { Wettbewerbsvorteil }\end{array}$} & \multicolumn{7}{|c|}{$\begin{array}{l}\text { BP, Handbuch, Coaching, Feedback, (Mentoring ab Markteintr } \\
\text { Geschäftsidee: Erfolgschancen ynd Kundennutzen }\end{array}$} & tt $) \rightarrow$ Strategie und \\
\hline $\begin{array}{l}\text { Umfeld } \\
\text { (Umfeld- } \\
\text { bezogener } \\
\text { Ansatz) }\end{array}$ & $\begin{array}{l}\text { Kooperationen } \\
\rightarrow \text { Gründungs- } \\
\text { einstellung } \\
\text { (Gründungs- } \\
\text { klima) }\end{array}$ & & & & \multicolumn{5}{|c|}{$\begin{array}{l}\text { Handbuch, Feedback, } \\
\text { Unterstützungsangebote } \\
\rightarrow \text { Vorbereitung Kunden, } \\
\text { Mitarbeiter, Kapital }\end{array}$} & \multicolumn{2}{|c|}{$\begin{array}{l}\text { Teilnahme (Reputations- } \\
\text { effekt), Nominierung und } \\
\text { Netzwerke } \\
\rightarrow \text { Sozialkapital } \\
\rightarrow \text { Mitarbeiter } \\
\rightarrow \text { Kunden } \\
\rightarrow \text { Eigen- und Fremdkapital } \\
\rightarrow \text { Grundstück, Geschäfts- } \\
\text { ausstattung, Material }\end{array}$} & $\begin{array}{l}\text { Netzwerke } \\
\rightarrow \text { Mitarbeiter } \\
\rightarrow \text { Kunden } \\
\rightarrow \text { Eigen- und } \\
\text { Fremdkapital } \\
\rightarrow \text { Geschäfts- } \\
\text { ausstattung, } \\
\text { Material }\end{array}$ \\
\hline \multirow{3}{*}{$\begin{array}{l}\text { Dimension 2= } \\
\text { Art der } \\
\text { Ressourcen- } \\
\text { quelle }\end{array}$} & \multirow{3}{*}{$\begin{array}{l}\text { Sensibilisie- } \\
\text { rungsphase }\end{array}$} & \multicolumn{10}{|c|}{ Gründungsprozess } & Bewährungs- \\
\hline & & & enphas & & \multicolumn{5}{|c|}{ Vorbereitungsphase } & \multicolumn{2}{|c|}{ Errichtungsphase } & \\
\hline & & $\begin{array}{c}\text { Gründ- } \\
\text { ungs- } \\
\text { interesse }\end{array}$ & $\begin{array}{l}\text { Ideen- } \\
\text { phase }\end{array}$ & $\begin{array}{c}\text { Grün- } \\
\text { dungs- } \\
\text { idee }\end{array}$ & $\begin{array}{c}\text { Informations- } \\
\text { und Orien- } \\
\text { tierungsphase }\end{array}$ & $\begin{array}{c}\text { Grün- } \\
\text { dungs- } \\
\text { ent- } \\
\text { schluss }\end{array}$ & $\begin{array}{c}\text { Machbar- } \\
\text { keitsunter- } \\
\text { suchung }\end{array}$ & $\begin{array}{l}\text { Machbar- } \\
\text { keits- } \\
\text { studie }\end{array}$ & $\begin{array}{c}\text { Ent- } \\
\text { wick- } \\
\text { lung BP }\end{array}$ & $\begin{array}{l}\text { Gewinnung von } \\
\text { Startressourcen }\end{array}$ & $\begin{array}{l}\text { Markt- } \\
\text { eintritt }\end{array}$ & Marktbearbeitung \\
\hline
\end{tabular}

Abb. l: Einfluss der Unterstützungsleistungen von BPW auf den Prozess der Ressourcenakkumulation 


\begin{tabular}{|c|c|c|}
\hline $\begin{array}{l}\text { Entwicklungs- } \\
\text { phase }\end{array}$ & $\begin{array}{l}\text { Ressourcen- } \\
\text { quelle }\end{array}$ & Proposition (P) \\
\hline Sensibilisierung & Allgemein & $\begin{array}{l}\text { P1: BPW tragen zu einer Verbesserung des Gründungsklimas } \\
\text { und damit zu einer Erhöhung des Gründungsinteresses bei. }\end{array}$ \\
\hline \multirow[t]{2}{*}{ Vorbereitung } & Gründerperson & $\begin{array}{l}\text { P2: Ein Rückgriff auf das Informationsangebot von BPW } \\
\text { zum Aufbau von Gründungswissen reduziert den Aufwand } \\
\text { der Informationssuche. } \\
\text { P3: (1) Das Handbuch, (2) die Workshops und (3) die Soft- } \\
\text { ware zur Finanzplanung fördern die Entwicklung der kri- } \\
\text { tisch-analytischen Planungsfähigkeiten der Gründer. } \\
\text { P4: Coaching-Gespräche helfen dabei, Lücken im Branchen- } \\
\text { und Gründungswissen zu kompensieren. }\end{array}$ \\
\hline & Gründungsidee & $\begin{array}{l}\text { P5: (1) Das Handbuch, (2) die Coaching-Gespräche und (3) } \\
\text { das Juroren-Feedback tragen mit konkreten Hinweisen zu ei- } \\
\text { ner Optimierung der Gründungsidee, bzw. der Umsetzungs- } \\
\text { konzeption bei oder führen zu dem Entschluss des frühzeiti- } \\
\text { gen Abbruch des Gründungsvorhabens. }\end{array}$ \\
\hline \multirow[t]{2}{*}{ Errichtung } & Gründerperson & $\begin{array}{l}\text { P6: Durch (1) Seminare und (2) das Informationsangebot von } \\
\text { BPW werden Wissenslücken im Gründungs- bzw. betriebs- } \\
\text { wirtschaftlichen Bereich geschlossen bzw. fehlende Kompe- } \\
\text { tenzen aufgedeckt. }\end{array}$ \\
\hline & Umfeld & $\begin{array}{l}\text { P7: (1) Datenbanken und (2) Netzwerke helfen bei der Her- } \\
\text { stellung von Kontakten zu Stakeholdern. }\end{array}$ \\
\hline \multirow[t]{2}{*}{ Bewährung } & Gründerperson & $\begin{array}{l}\text { P8: Das Mentoring hilft Gründern mit Ratschlägen bei der } \\
\text { Überwindung von Hindernissen in der Bewährungsphase. }\end{array}$ \\
\hline & Umfeld & $\begin{array}{l}\text { P9: Aus Kontakten des aufgebauten Netzwerks ergeben sich } \\
\text { konkrete Verträge bzw. der Gewinn von externen Ressourcen. }\end{array}$ \\
\hline \multirow[t]{2}{*}{$\begin{array}{l}\text { Über alle } \\
\text { Phasen hinweg }\end{array}$} & Gründerperson & $\begin{array}{l}\text { P10: Eine Teilnahme an BPW erhöht die Motivation der } \\
\text { Gründer und senkt ihre Unsicherheit. }\end{array}$ \\
\hline & Allgemein & $\begin{array}{l}\text { P11: Durch eine Teilnahme an BPW steigen die Chancen } \\
\text { eines erfolgreichen Aufbaus und Betriebs des neuen Unter- } \\
\text { nehmens. }\end{array}$ \\
\hline
\end{tabular}

Tab. 2: Propositionen zum Einfluss der Unterstützungsleistungen von BPW auf den Prozess der Ressourcenakkumulation derungen im Unternehmensaufbau und -betrieb benötigen (,Entrepreneurship Education“). Gleichzeitig unterstützen BPW den Zugang zu externen Ressourcen direkt oder indirekt. Dieser Unterstützungsansatz kann dem stärker individualisierten „Entrepreneurial Support“ zugeordnet werden.

\section{Fazit und Implikationen für die Entrepreneurship-Forschung und -Praxis}

BPW können durch die Ausübung einer Selektions- und Förderfunktion zu dem Gründungs- und Etablierungserfolg ausgewählter Start-up Projekte beitragen. Die empirischen Ergebnisse zum Business Planning zeigen, dass die Forderung eines BP hauptsächlich der Selektionsfunktion der BPW dient. Der Nutzen der BP-Erstellung für den Gründungs- und Etablierungserfolg ist von Gestaltungsund Kontextfaktoren abhängig. Für eine umfassendere empirische Fundierung des entwickelten Bezugsrahmens müssten in einem nächsten Schritt die Unterstützungsangebote der BPW hinsichtlich ihrer Wirkung auf gründungsrelevante personen-, betriebs- und umfeldbezogene Ressourcen getestet werden. Wie bei dem BP-Element könnten auch hier Moderator- und Mediatorvariablen (z. B. Ausrichtung des BPW auf eine bestimmte Industrie, Vorwissen und Erfahrung der Gründer, allgemeine wirtschaftliche Situation) eine wichtige Rolle spielen. 
In Bezug auf die ausstehende empirische Überprüfung der Propositionen kann zunächst auf bereits existierende Daten zurück gegriffen werden. So weisen beispielsweise die Überlebensquoten der aus den BPW resultierenden Unternehmen auf eine Verbesserung der von dem Bund Deutscher Unternehmer ermittelten, generellen Überlebensquote von $40 \%$ hin. Bestehende empirische Untersuchungen mit Teilnehmern von BPW untersuchen insbesondere den Unterstützungsbedarf der Gründer sowie deren Zufriedenheit mit den Unterstützungsleistungen (vgl. Dippe/Müller, 2005; Kerlen/Buhr, 2007a; Kerlen/Buhr, 2007b, Universität Witten/Herdecke/Loyola University Chicago, 2008). Schlussfolgerungen hinsichtlich des BPW-Nutzens auf Basis der Teilnehmerzufriedenheit sind jedoch nur beschränkt möglich, da die Beurteilung der Zufriedenheit stark von den persönlichen Erwartungen abhängt. Die Unzulänglichkeiten der untersuchten empirischen Studien zeigen den Bedarf an weiteren Forschungen zum Nutzen von BPW auf.

Aus den Ergebnissen des vorliegenden Beitrages lassen auch sich Implikationen für die Ausrichter von BPW hinsichtlich der Gestaltung von Wettbewerben, für die Teilnehmer als auch für Stakeholder wie staatliche und private Kapitalgeber ableiten. Für die Ausrichter von BPW ergäbe sich bei empirischer Bestätigung des theoretisch fundierten Konzepts, d. h. der Selektionsfunktion und Verbesserung der Ressourcenausstattung von erfolgsträchtigen, jungen Unternehmen, insgesamt eine Legitimationsgrundlage. Damit der positive Nutzen von BPW seine größte Wirkung erlangt, sollte jedoch Abstand von einem „Onesize-fits-all“"Design bei der Planungsgestaltung genommen werden. In Handbüchern und bei Workshops und Coaching sollten verstärkt nicht nur Informationen zu den Planungsinhalten, sondern auch zu dem Planungsprozess sowie bezüglich der Verwendung der Planungsergebnisse gegeben werden. Derzeitig verwendete Handbücher zur BP-Erstellung, Seminarunterlagen und damit die Anforderungen an den Planungsprozess und -inhalt sind meist stark standardisiert. Dies erhöht zwar einerseits die Vergleichbarkeit der Beiträge für die Jury und erleichtert den Zugang zu Kapitalgebern. Andererseits besteht der Verdacht, dass dabei der Planungsaufwand den potenziellen Planungsnutzen bei vielen Gründungsvorhaben übersteigt.

Zur Berücksichtigung des Individualisierungsbedarfs bei der BP-Erstellung sind zudem die Anforderungen zur Nominierung zu flexibilisieren. So könnte verlangt werden, dass die Teilnehmer in einer mündlichen Präsentation zunächst die Planungsergebnisse problemorientiert darstellen. Darüber hinaus könnte ein externes Dokument mit zumindest den Elementen Executive Summary, Produkt, Kundennutzen sowie Finanzplanung in schriftlicher Form verlangt werden. Ziel dieser Gestaltung wäre es, den externen und internen Nutzen des Business Planning zu vereinen und dabei Raum für individuelle Planungsbedingungen zu lassen.
BPW üben durch die in diesem Beitrag theoretisch hergeleitete Selektions- sowie Förderfunktion im Allgemeinen eine positive Wirkung auf Unternehmensgründungen aus. Vor diesem Hintergrund sollten sie Zielobjekt staatlicher Unterstützung sein. Allerdings sollten die Fördermaßnahmen für die Wettbewerbsteilnehmer auch individualisierte Komponenten enthalten, um möglichen Dysfunktionen des Business Planning entgegenzuwirken. Für Kapitalgeber müssten sich die geförderten Unternehmen, sofern sie dem Selektionsprozess des Wettbewerbs standgehalten haben, dann als lukrative Investitionsobjekte erweisen.

\section{Literatur}

Baker, T., R. Nelson, Creating something from nothing: Resource construction through entrepreneurial bricolage, in: Administrative Science Quarterly, Vol. 50 (2005), S. 329-366.

Brinckmann, J., D. Grichnik, D. Kapsa, Should entrepreneurs plan or just storm the castle? A meta-analysis on contextual factors impacting the business planning-performance relationship in small firms, in: Journal of Business Venturing, Vol. 25 (2010), S. 24-40.

Castrogiovanni, G. J., Pre-startup planning and the survival of new small businesses: Theoretical linkages, in: Journal of Management, Vol. 22 (1996), S. 801-822.

Chwolka, A., M. Raith, Der Businessplan als institutionalisiertes Informationssystem im Gründungsprozess, in: H. Gischer, P. Reichling, T. Spengler, A. Wenig (Hrsg.), Transformation in der Ökonomie. Wiesbaden 2008, S. 347-370.

Dippe, A., T. Müller, Unternehmensgründungen und ihre Unterstützung durch Gründungswettbewerbe, in: H. G. Gemünden, S. Salomo, T. Müller (Hrsg.), Entrepreneurial excellence, Wiesbaden 2005, S. 293-328.

Kerlen, C., R. Buhr, Erfolgsfaktoren im Bereich Multimedia. Evaluation des Gründerwettbewerbs Mit Multimedia erfolgreich starten - Arbeitspapier Nr. 2/2007. 2007a. In: http://www.gruen derwettbewerb.de/Downloads/download/erfolgsfaktoren.pdf (23.06.2008).

Kerlen, C., R. Buhr, Bilanz des Gründungswettbewerbs Mit Multimedia erfolgreich starten. Evaluation des Gründerwettbewerbs Mit Multimedia erfolgreich starten - Arbeitspapier Nr. 3/2007, 2007b. In: http://www.gruenderwettbewerb.de/Downloads/down load/bilanz2005-2006.pdf (24.06.2008).

Knyphausen-Aufseß, D. zu, S. Goodwin, Die Gestaltung von Gründungswettbewerben, in: Zeitschrift für Management, Vol. 4 (2009), S. 131-152.

Locke, E., G. Latham, A theory of goal setting and task performance, New Jersey 1990.

McGrath, R., I. MacMillan, Discovery-driven planning, in: Harvard Business Review, Vol. 73, Nr. 4 (1995), S. 44-53.

Mellewigt, T., F. Schmidt, I. Weller, Stuck in the Middle - Eine empirische Untersuchung zu Barrieren im Vorgründungsprozess, in: Zeitschrift für Betriebswirtschaft, Special Issue Nr. 4 (2006), S. 93-115.

Mintzberg, H., Die strategische Planung - Aufstieg, Niedergang und Neubestimmung. München, Wien 1995.

Szyperski, N., K. Nathusius, Probleme der Unternehmensgründung, Stuttgart 1977.

Universität Witten/Herdecke/Loyola University Chicago, Strategischer Planungsprozess von Unternehmensgründern (2008), in: http://www.mbpw.de/index.php?id=157.

Van de Ven, A., R. Hudson, D. Schroeder, Designing new business startups: Entrepreneurial, organizational, and ecological considerations, in: Journal of Management, Vol. 10 (1984), S. 87-107. 